\title{
Interview Functional Independence Measure score: self-reporting as a simpler alternative to multidisciplinary functional assessment
}

\author{
Shaji Jose Vadassery ${ }^{1}$, MRCP, FAMS, Keng He $\underline{K o n g}^{2}$, MRCP, FAMS, Wai Mun Lorraine $\underline{\mathrm{Ho}}^{3}$, MBBS, \\ Aruni Seneviratna ${ }^{4}$, MBBS, MPH
}

\begin{abstract}
INTRODUCTION The Functional Independence Measure (FIM) is a validated, objective assessment of functional status. It is widely used in rehabilitation centres but may not be practical for all patients due to time and/or personnel constraints. Studies show positive and negative agreement on self-reported FIM scores for patients with spinal cord injuries and amputees. We tested the validity of the self-reported FIM motor score among stroke patients.

METHODS We conducted a prospective double-blind comparative study of patient self-reporting against multidisciplinary assessment, using the standard FIM algorithm. All eligible stroke patients $(n=47)$ admitted to our rehabilitation centre were included. 33 patients were included in the final analysis.

RESULTS There was substantial agreement on overall FIM motor score between patient self-reporting and multidisciplinary assessment (intraclass correlation coefficient [ICC] 0.651, 95\% confidence interval 0.404-0.811). The scores of individual motor items also showed fair-to-good agreement (ICC range 0.431-0.618), except for eating, grooming, bathing and dressing of the lower body (ICC < 0.400).

CONCLUSION There was no FIM assessment for 14 (29.8\%) patients, highlighting the need for alternative assessment tools. Discrepancies in some scores could be due to patients' ignorance of their own limitations or feelings of embarrassment about reporting. Our results may not be valid for patient populations with cognitive or communication deficits. However, a modest agreement between patient self-reporting and multidisciplinary assessment of FIM motor score was demonstrated. Although patients tend to overrate their performance, self-reported FIM motor scores could be an alternative in situations where multidisciplinary FIM assessment is difficult.
\end{abstract}

Keywords: functional status, motor score, self report, stroke

\section{INTRODUCTION}

During rehabilitation, functional assessment is important to not only accurately assess patients' functional improvement but also to help with prognostication, individualisation of patient care, quality assurance and national healthcare planning. The Functional Independence Measure (FIM) is a validated, objective assessment of functional status that is commonly used in rehabilitation centres. As it allows direct observation of patients and the performance-based assessments are done by multidisciplinary teams - including doctors, therapists and nurses - FIM is considered the gold standard for functional assessments. However, multidisciplinary measurement of FIM scores is time-consuming and laborious, and hence may not be feasible for all patients. There is thus a need for an alternative and reliable method that can be easily delivered, which could also improve follow-up assessments and aid in future research.

Studies on the validity of patient self-reported FIM (FIM-SR) have shown both positive and negative agreement. A study by Massedo et al on FIM-SR assessment among patients with spinal cord injuries and chronic pain, as well as amputees, showed reliable results in the FIM-SR motor scale and total FIM-SR score, but poor agreement in cognitive score. ${ }^{(1)}$ The authors also found poor agreement of scores in bathing (63\%) and dressing (64\%). Another study among elderly patients with hip fractures showed comparable mean FIM ratings between trained personnel and the multidisciplinary team (84.3 vs. 80.5; $p<0.001$ ), with an intraclass correlation coefficient (ICC) of 0.74. ${ }^{(2)}$ Studies on stroke patients ${ }^{(3)}$ and patients with spinal cord injuries ${ }^{(4)}$ also showed comparable ratings. In another study, two statistically significant factors found to affect the outcome among hospitalised elderly patients were: (a) cognitive impairment; and (b) decline in activities of daily living (ADLs) from pre-hospitalisation levels. ${ }^{(5)}$ Major limitations of these earlier studies included their small sample sizes and the restriction of study criteria to include patients with only non-stroke-related functional impairments. To the best of our knowledge, the only other study done among stroke patients compared telephone FIM ratings; moreover, the assessment was done by a registered nurse rather than a multidisciplinary team. ${ }^{(3)}$ Therefore, our study tested the validity of self-reported FIM motor scores in stroke patients against scores obtained through multidisciplinary assessment.

\section{METHODS}

This was a prospective, double-blind study comparing patient selfreporting of the motor subset of the FIM against multidisciplinary

${ }^{1}$ Department of General Medicine, ${ }^{2}$ Department of Rehabilitation Medicine, Tan Tock Seng Hospital, ${ }^{3}$ Yong Loo Lin School of Medicine, National University of Singapore, ${ }^{4}$ Clinical Research Unit, Tan Tock Seng Hospital, Singapore

Correspondence: Dr Shaji Jose Vadassery, Consultant, Department of General Medicine, Tan Tock Seng Hospital, 11 Jalan Tan Tock Seng, Singapore 308433. shaji_jose_vadassery@ttsh.com.sg 
Table I. Summary of Functional Independence Measure (FIM) motor score assessments.

\begin{tabular}{|c|c|c|c|c|}
\hline \multirow[t]{2}{*}{ Variable } & \multicolumn{2}{|c|}{ Mean \pm SD } & \multirow[t]{2}{*}{ ICC (95\% CI) } & \multirow[t]{2}{*}{ Agreement* } \\
\hline & Multidisciplinary assessment & Patient self-reporting & & \\
\hline Overall FIM motor score & $66.91 \pm 13.07$ & $72.45 \pm 11.85$ & 0.651 (0.404 to 0.811$)$ & Good \\
\hline \multicolumn{5}{|l|}{ Individual FIM motor item } \\
\hline Eating & $6.48 \pm 0.38$ & $6.82 \pm 0.39$ & $-0.123(-0.441$ to 0.223$)$ & Poor \\
\hline Grooming & $5.82 \pm 1.26$ & $6.39 \pm 1.46$ & $0.026(-0.313$ to 0.350$)$ & Poor \\
\hline Bathing & $4.94 \pm 1.32$ & $6.15 \pm 1.28$ & $0.184(-0.162$ to 0.491$)$ & Poor \\
\hline Upper body dressing & $5.67 \pm 1.24$ & $6.33 \pm 1.16$ & 0.431 (0.090 to 0.658 ) & Fair \\
\hline Lower body dressing & $4.94 \pm 1.30$ & $5.61 \pm 1.90$ & 0.370 (0.039 to 0.628$)$ & Poor \\
\hline Toileting & $5.12 \pm 1.36$ & $5.52 \pm 1.86$ & 0.472 (0.163 to 0.698$)$ & Fair \\
\hline Bladder & $5.30 \pm 1.42$ & $5.36 \pm 2.04$ & 0.488 (0.183 to 0.709$)$ & Fair \\
\hline Bowel & $5.27 \pm 1.57$ & $5.76 \pm 1.64$ & $0.536(0.245$ to 0.740$)$ & Fair \\
\hline Wheelchair transfer & $5.06 \pm 1.22$ & $5.61 \pm 1.20$ & 0.545 (0.256 to 0.745$)$ & Fair \\
\hline Toilet transfer & $5.06 \pm 1.09$ & $5.39 \pm 1.50$ & 0.465 (0.154 to 0.694$)$ & Fair \\
\hline Shower transfer & $4.97 \pm 1.07$ & $5.09 \pm 1.33$ & 0.563 (0.281 to 0.757$)$ & Fair \\
\hline Walking & $4.70 \pm 1.26$ & $4.85 \pm 1.42$ & $0.586(0.312$ to 0.771$)$ & Fair \\
\hline Stair climbing & $3.58 \pm 1.58$ & $3.58 \pm 1.89$ & 0.618 (0.357 to 0.791$)$ & Good \\
\hline
\end{tabular}

*ICC agreement categories were poor (range 0.00-0.40), fair (range 0.41-0.60), good (range 0.61-0.74) and excellent (range 0.75-1.00). Cl: confidence interval; ICC: intraclass correlation coefficient; SD: standard deviation

assessment. The cognitive and communication domains of FIM were excluded during both assessments because it is difficult to obtain accurate self-reports of these items. The FIM motor score is also a measure of independence in mobility and performance of ADLs. The study was approved by the institutional ethics review board.

We included all patients with a diagnosis of new stroke admitted to the Department of Rehabilitation Medicine in Tan Tock Seng Hospital, a tertiary care hospital in Singapore, from October 2013 to May 2014. Patients who were premorbidly dependent in their ADLs as well as those who had aphasia, depression, cognitive impairment, chronic pain or acute medical deterioration were excluded. Among the 97 patients admitted, 47 patients satisfied the eligibility criteria. Another 14 patients were also excluded, as their FIM assessments had not been performed by the multidisciplinary team. The final analysis sample of 33 was achieved within the available time and manpower constraints.

Multidisciplinary assessment and patient self-reporting of the 13 individual motor items based on the standard FIM scoring algorithm were recorded during the last week prior to discharge. Patient reporting was documented by an interviewer, a fourth-year medical student, who was certified to administer the FIM. Both the multidisciplinary team and interviewer were blinded to each other's assessment. The individual scores were then summed up to obtain the overall FIM motor score of each patient. We compared the individual scores and overall FIM motor scores obtained during the assessments by the multidisciplinary teams and selfreporting patients. Bland-Altman plot and ICC were computed for the overall FIM motor score as well as the 13 individual items to assess agreement and consistency between patient self-reporting and multidisciplinary assessment. ${ }^{(6)}$ Statistical analyses were performed using IBM SPSS Statistics version 20.0 (IBM Corp, Armonk, NY, USA).

\section{RESULTS}

The mean age of the patients was 61 years and they were ethnically Chinese, Malay and Indian. We found good agreement for overall FIM motor scores between patient self-reporting and multidisciplinary assessment, with an ICC of 0.651 (95\% confidence interval [CI] 0.404-0.811) (Table I). Scores of the 13 individual motor items also showed fair-to-good inter-rater reliability, with ICC in the range of 0.431-0.618, except for eating, grooming, bathing and dressing of the lower body (ICC $<0.400$ ). Bland-Altman analysis (Fig. 1) found a mean difference in overall FIM motor score of 5.5 (95\% Cl 2.3-8.8); the limits of agreement were -12.5 to 23.6. Except for one, all other observations were spread within 1.96 standard deviations.

\section{DISCUSSION}

The present study evaluated the validity of self-reported FIM motor scores in stroke patients as an easier alternative to FIM scoring by a multidisciplinary team. For overall FIM motor scoring, good agreement was seen between multidisciplinary assessment and patient self-reporting.

Self-reporting by patients generally produced higher ratings than assessment by the multidisciplinary team (Table I). The higher rating given by patients for ADLs, such as eating, bathing, dressing of the lower body and grooming, could be partly due to the patients' embarrassment about reporting them, as suggested in other studies.(2) The discrepancy may also be due to ignorance about their own limitations while being an inpatient, as patients may perform these activities with assistance at the rehabilitation centre. The post-discharge assessment may provide a more accurate evaluation of these items. Previous studies on patients discharged home have shown the reliability of FIM scores obtained through telephone interviews. ${ }^{(3)}$ 


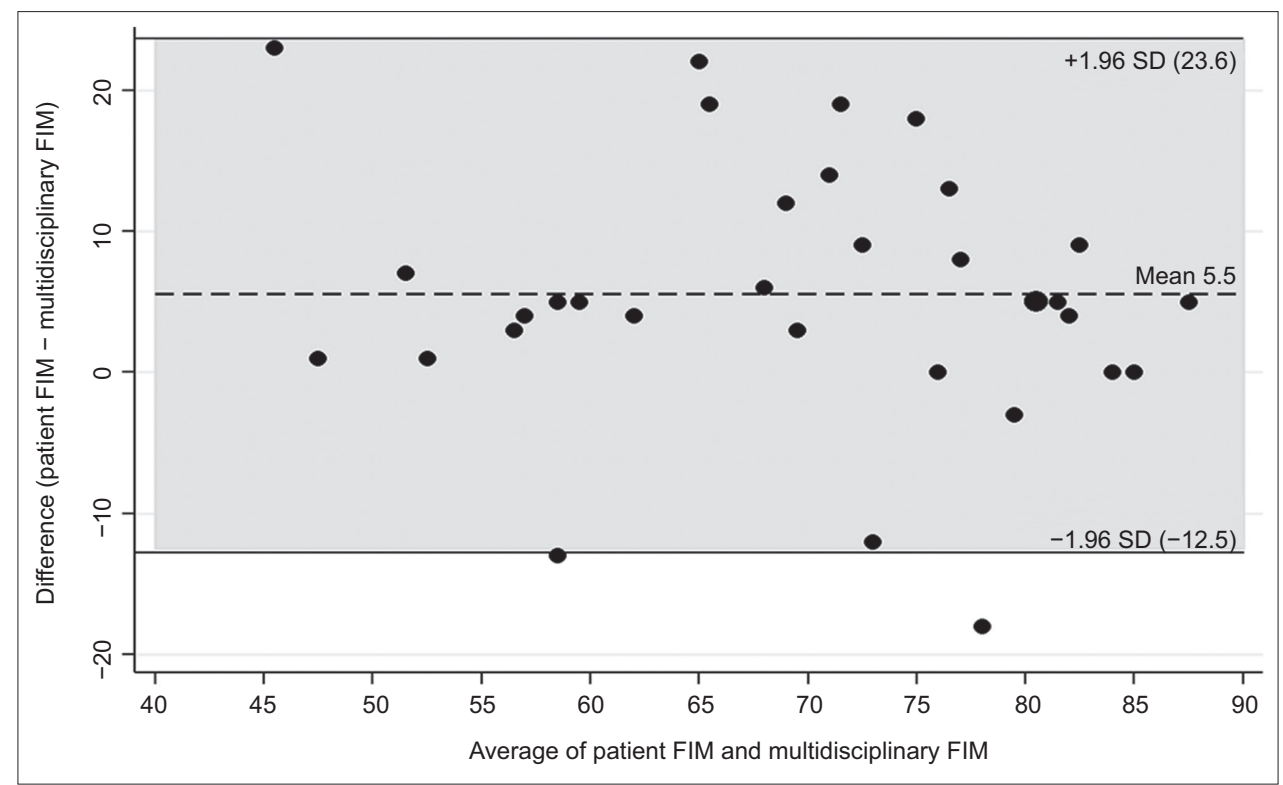

Fig. 1 Bland-Altman plot graph shows the agreement between multidisciplinary assessment and patient self-reporting of Functional Independence Measure (FIM) motor scores. The grey shaded area represents the 95\% limits of agreement. SD: standard deviation

The limits of agreement between self-reported and multidisciplinary team FIM scores were between -12.5 and 23.6. Among stroke patients, the reported minimal clinically important difference in FIM motor score was 17 points, while the moderate clinically important difference was 22 points. ${ }^{(7}$ We believe that the observed error limits in this study are within accepted clinical limits and are unlikely to lead to clinical consequences.

As the multidisciplinary team did not perform the FIM assessment for $14(29.8 \%)$ out of 47 patients who met the inclusion criteria, these patients had to be excluded from the study. We assumed this was partly due to manpower and/or time constraints, and expect this to be commonplace in rehabilitation centres. This finding, however, underscored the need for alternative assessment tools, such as interviewer-based or patient self-reported scoring systems, which can be reliably used for inpatients in rehabilitation centres when multidisciplinary FIM assessment may be difficult.

Simple alternative modalities for functional status assessments are useful for long-term follow-up of these patients following discharge. If FIM could be reliably assessed by a trained interviewer or via telephone, resources and medical personnel would be better utilised. It would also help to address the key shortcoming of patients who are lost to follow-up after discharge, as not all discharged patients return for outpatient reviews.

Our study was not without limitations. We only compared the FIM motor scores of a group of stroke patients who had completed rehabilitation. Hence, the applicability of our data is limited to assessments of functional recovery and coping. The results may not be valid for other patient populations, such as patients with cognitive or communication deficits, or those with low functional capacity. Although there was agreement in functional mobility scores, ratings of basic ADLs (e.g. eating and grooming) showed poor agreement, which concurred with the results of previous studies. ${ }^{(3)}$

In conclusion, our study demonstrated modest agreement between patient self-reporting and multidisciplinary assessment of FIM motor scores as a measure of functional ability in stroke patients. Although patients tend to overrate their performance, our results suggest that patient self-reported FIM motor scores could be considered as an alternative in situations where a full multidisciplinary assessment may not be feasible. Future studies could also ascertain the robustness of such patient self-reporting in the outpatient setting.

\section{REFERENCES}

1. Massedo Al, Hanley M, Jensen MP, Ehde D, Cardenas DD. Reliability and validity of a self-report FIM (FIM-SR) in persons with amputation or spinal cord injury and chronic pain. Am J Phys Med Rehabil 2005; 84:167-76; quiz 177-9, 198.

2. Young Y, Fan MY, Hebel JR, Boult C. Concurrent validity of administering the functional independence measure (FIM) instrument by interview. Am J Phys Med Rehabil 2009; 88:766-70.

3. Smith PM, Illig SB, Fiedler RC, Hamilton BB, Ottenbacher KJ. Intermoda agreement of follow-up telephone functional assessment using the Functional Independence Measure in patients with stroke. Arch Phys Med Rehabil 1996; 77:431-5.

4. Hoenig H, Branch LG, McIntyre L, Hoff J, Horner RD. The validity in persons with spinal cord injury of a self-reported functional measure derived from the functional independence measure. Spine (Phila Pa 1976) 1999; 24:539-44.

5. Sager MA, Dunham MC, Schwantes A, et al. Measurements of activities of daily living in hospitalized elderly: a comparison of self-report and performance-based methods. J Am Geriatr Soc 1992; 40:457-62.

6. Cicchetti DV. Guidelines, criteria, and rules of thumb for evaluating normed and standardised assessment instruments in psychology. Psychol Assess 1994; 6:284-90.

7. Beninato M, Gill-Body KM, Salles S, Stark PC, Black-Schaffer RM, Stein J. Determination of the minimal clinically important difference in the FIM instrument in patients with stroke. Archives of physical medicine and rehabilitation 2006; 87:32-9. 